\title{
Utilizing research into electrical double layers as a basis for the development of label-free biosensors based on nanomaterial transistors
}

This article was published in the following Dove Press journal:

Nanobiosensors in Disease Diagnosis

30 December 2015

Number of times this article has been viewed

\author{
Kenzo Maehashi' \\ Yasuhide Ohno ${ }^{2}$ \\ Kazuhiko Matsumoto ${ }^{3}$ \\ 'Institute of Engineering, Tokyo \\ University of Agriculture and \\ Technology, Koganei, Tokyo, \\ ${ }^{2}$ Institute of Technology and Science, \\ The University of Tokushima, \\ Tokushima, ${ }^{3}$ The Institute of Scientific \\ and Industrial Research, Osaka \\ University, Ibaraki, Osaka, Japan
}

\begin{abstract}
Label-free detection of biomolecules has attracted great attention in a lot of life science fields such as genomics, clinical diagnosis, and practical pharmacy. In recent years, for early detection and medical treatment of serious disease, hand-held biosensors for home medical care systems have been intensely developed. In this article we review label-free biosensors based on nanomaterial transistors in which silicon nanowires, carbon nanotubes, and graphene are used as channels. Such nanomaterial-based transistors have high potential for fabrication of label-free biological sensors with high sensitivity. In this review we introduce the detection mechanism of biomolecules using transistors, and show that an electrical double layer in a solution acts as a thin insulator with dielectric constant in transistor-based biosensors. Moreover, we discuss the effect of the electrical double layers in a solution on sensitivity of nanomaterial transistor-based biosensors using the example of label-free electrical detection of biomolecules and fabrication technology of biosensors based on nanomaterial transistors.
\end{abstract}

Keywords: label-free biosensors, Si nanowires, carbon nanotubes, graphene, field-effect transistors, electrical double layers

\section{Introduction}

Over the past decade, highly sensitive detection of biological or chemical molecules has attracted much attention for various applications, including clinical diagnostics, environmental testing, food analysis, and bioterrorism detection technologies. ${ }^{1-6}$ In recent years the development of hand-held biosensors for home medical care systems has been strongly required for effective early detection and medical treatment of serious disease. Currently, detection of biological molecules generally depends on optical methods with a fluorescent material, such as enzyme-linked immunosorbent assay. Although these methods have high sensitivity and selectivity, they need expensive apparatus and highly professional knowledge and techniques for the labeling process. To realize hand-held biosensors for home medical care systems, label-free electrical detection of chemical and biological species is worthy of notice because it is simpler and less expensive, requires less energy than conventional methods, and can be applied to miniaturization of biosensors.

Several types of label-free electrical biosensors have been developed, such as amperometric biosensors, electrochemical impedance biosensors, and field-effect transistor (FET)-based biosensors. In electrochemical amperometric sensing, current is monitored between working and counter electrodes when electroactive biomolecules in a solution are oxidized or reduced. ${ }^{7,8}$ As a result, biomolecules can be detected using amperometric biosensors. On the other hand, for impedance biosensors, solution 
impedance at interfaces is measured using a different frequency. ${ }^{9,10}$ When target molecules are bound to receptors, the impedance will be changed.

In this article we review label-free potentiometric biosensors based on nanomaterials such as silicon ( $\mathrm{Si}$ ) nanowires, carbon nanotubes (CNTs), and graphene. As compared with three-dimensional Si FETs, lowdimensional nanomaterial FETs are expected for fabricating highly sensitive biological sensors. In this review we focus on the detection mechanism of target molecules, discuss the effect of electrical double layers in a solution, and show that the Debye length affects the sensitivity of the sensors. Then, we introduce structures and fabrication techniques of label-free electrical biosensors based on nanomaterial FETs.

\section{Detection mechanism of biomolecules using FETs}

In general, three types of electrodes are utilized for FET-based sensing: source, drain, and gate electrodes. A semiconducting channel such as nanowires, CNTs, and graphene is bridged between the source and drain electrodes. A reference electrode such as silver $(\mathrm{Ag}) /$ silver chloride $(\mathrm{AgCl})$ is used as the gate electrode to minimize environmental effects in a solution. ${ }^{11}$ For specific sensing of target molecules, the channel is functionalized with receptors that comprise any organic or inorganic material. For example, an antibody (receptor) is reacted to an antigen (target). The FET works as a transducer and the chemical information of target molecules can be converted to electrical signals.

Figure 1A shows a simple detection mechanism for biomolecules using an FET that has a p-type characteristic. After target biomolecules are bound to receptors on the channel, the band of the channel is modulated. When the target molecules have negative charges in a solution, holes accumulate in the channel, resulting in an increase in the source-drain current. On the other hand, when positively charged molecules react to the receptors, the hole density in the channel is depleted. As a result, a reduction in source-drain current is observed. Therefore, FETs work as transducers and electrically detect biomolecules. In threedimensional Si FETs, adsorption of biomolecules induces modulation of a band at only a thin region near the channel surface. In contrast, accumulation or depletion of carriers for entire regions of nanomaterial channels occurs for lowdimensional nanomaterial FETs after target molecules are bound on the surface. For this reason, the nanomaterial FETs are expected for fabricating highly sensitive biological sensors.
Kim et al ${ }^{12}$ reported detection of prostate-specific antigen (PSA) using n-type Si nanowire FETs (Figure 1B). They used two kinds of buffer solutions at $\mathrm{pH} 6.0$ and 7.8. As the isoelectric point (pI) of PSA is 6.9, PSA has positive and negative charges in the buffer solutions at $\mathrm{pH} 6.0$ and 7.8, respectively. The time dependence of the conductance exhibited the decrease and increase in the conductance of the $\mathrm{Si}$ nanowire FETs in the buffer solutions at $\mathrm{pH} 7.8$ and 6.0 after introduction of PSA (Figure 1B), respectively. For graphene FETs, bovine serum albumin (BSA) was detected in buffer solutions at $\mathrm{pH} 4.0$ and 6.8 (Figure 1C). ${ }^{13}$ The graphene FETs have ambipolar characteristics. ${ }^{14}$ The measurements were carried out in the p-type characteristic region. Owing to the $\mathrm{pI}(5.6)$ of BSA, in the buffer solution at $\mathrm{pH} 6.8$ the drain current increased after injection of the BSA. On the other hand, the drain current decreased for the buffer solution at $\mathrm{pH}$ 4.0. Thus, nanomaterial-based FETs can detect both positive and negative charges of biomolecules.

\section{Electrical double layer}

Typical Si FETs act in the atmosphere, and their performance depends on the variety and thickness of the gate insulators. In contrast, FET-based biosensors work in a solution, and the electrical double layer is an important factor that affects the sensing performance (Figure 2A). It was reported that the comparison of transfer characteristics in the graphene FET against back gate voltage in vacuum and electrolyte gate voltage in an electrolyte was demonstrated. ${ }^{15}$ The device has a silicon dioxide $\left(\mathrm{SiO}_{2}\right)(\sim 280 \mathrm{~nm})$ layer and operates in a solution of phosphate buffer solution (PBS) $(10 \mathrm{mM})$. The measurements revealed that the device has an ambipolar characteristic in a vacuum (red line) or in an electrolyte (blue line) and that the transconductance of the electrolytegated graphene FET is more than 100-fold larger than that of the back-gated graphene FET in vacuum (Figure 2B and C). Thus, this result is because the electrical double layer is formed on the graphene channel and the layer acts as a thin insulator with dielectric constant after applying the gate voltage through the reference electrode (Figure 2A).

The thickness of the electrical double layer is described as the Debye length. ${ }^{16}$ That is simply defined as the distance from the channel surface. After binding events occur, excess charges inside the Debye length of a solution can affect a modulation of the band structure in the channel (Figure 2A). As a result, changes in the drain current can be monitored. However, if target molecules react to receptors outside the electrical double layer, the excess charges are screened by ions of the buffer solution, resulting in little changes in the drain current. The Debye length $\left(\lambda_{\mathrm{D}}\right)$ in a 


\section{A}

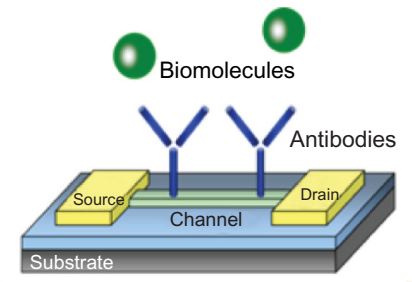

P-type FET

Negatively charged

Biomolecules
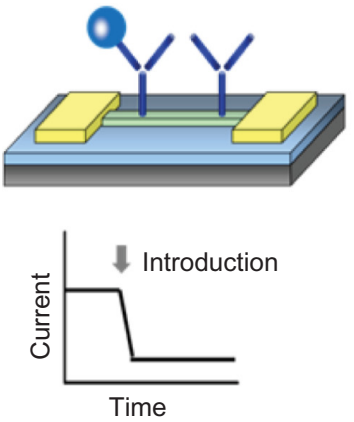

Positively charged Biomolecules
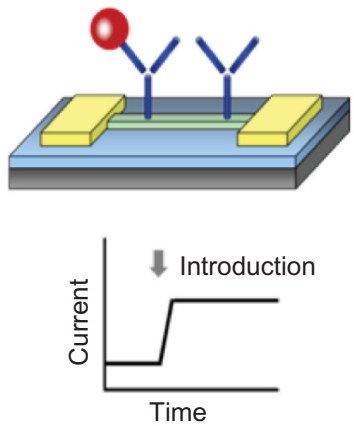

B

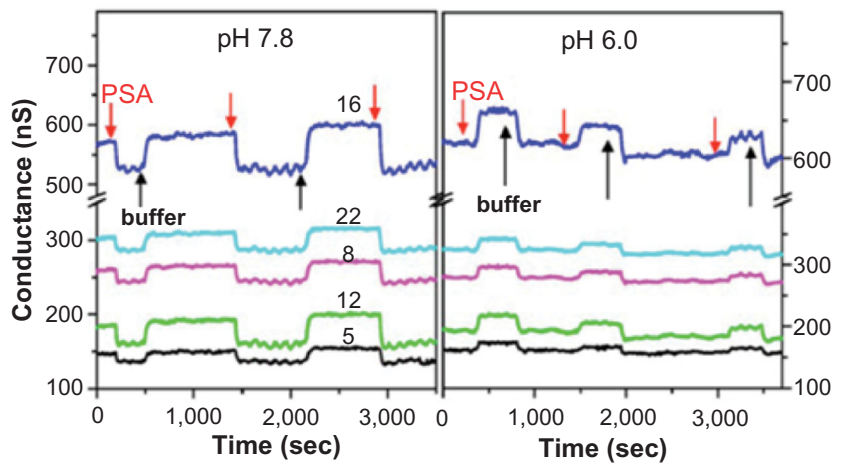

C
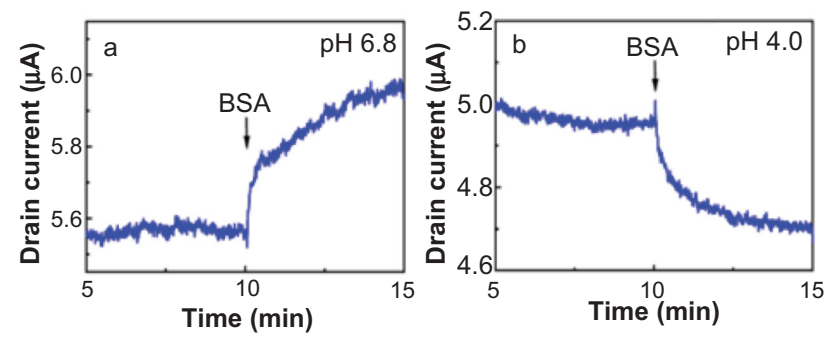

Figure I Biosensors using field-effect transistors (FETs).

Notes: (A) Detection mechanism for biomolecules using field-effect transistors that have p-type characteristics. When the target molecules have negative charges, the source-drain current increases. On the other hand, when positively charged molecules react to the receptors, a reduction in source-drain current is observed. (B) Detection of prostate-specific antigen (PSA) using n-type silicon ( $\mathrm{Si}$ ) nanowire FETs in buffer solutions at pH 7.8 (left side) and 6.0 (right side). ${ }^{13}$ (C) Detection of bovine serum albumin (BSA) using graphene FETs in buffer solutions at pH 4.0 (left side) and 6.8 (right side). Reproduced with permission from Kim A, Ah CS, Yu HY, et al. Ultrasensitive, labelfree, and real-time immunodetection using silicon field-effect transistors. Appl Phys Lett. 91:10390I. Copyright 2007, AIP Publishing LLC. ${ }^{12}$ Reproduced with permission from Okamoto S, Ohno Y, Maehashi K, et al. Immunosensors based on graphene field-effect transistors fabricated using antigen-binding fragment. Jpn J Appl Phys. 20I2;5 I:06FD08. Copyright 2012 The Japan Society of Applied Physics. ${ }^{13}$

solution can be approximately calculated using an equation given by:

$$
\lambda_{\mathrm{D}}=0.32(I)^{-1 / 2}
$$

where $I$ is the ionic strength of a buffer solution. Typical sizes of antibody and antigen vary $10-15 \mathrm{~nm}$ and
5-10 nm, respectively. ${ }^{17,18}$ For this reason, to detect the antigen-antibody reaction, a Debye length of more than $15-25 \mathrm{~nm}$ is required. As shown in Equation 1, the Debye length varies as the inverse square root of the ionic strength. Thus, buffer solution with a concentration of less than $1 \mathrm{mM}$ is needed for detection of the antigen-antibody reaction. Stern et $\mathrm{al}^{19}$ demonstrated an effect of buffer solution concentration 

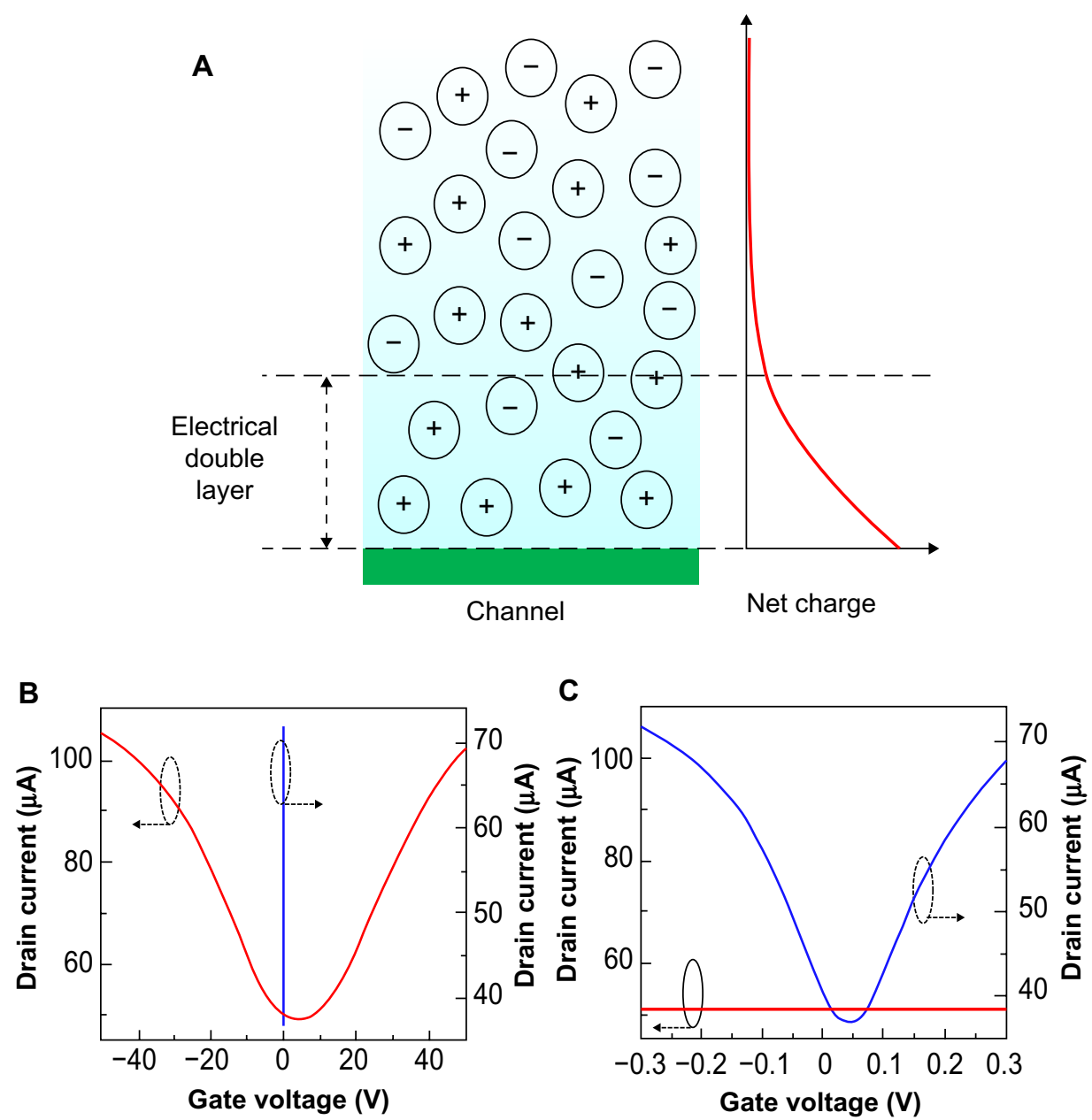

Figure 2 Electrical double layer in field-effect transistors (FETs).

Notes: (A) Schematic illustration of electrical double layer in a solution. Positive charges are accumulated near a channel. (B) Transfer characteristics in the graphene field-effect transistor against back gate voltage in vacuum (red line) and electrolyte gate voltage in a solution (blue line). ${ }^{15}$ (C) shows the enlarged view of (B). Reprinted from Sensors Actuators B: Chem. Vol 187.Maehashi K, Sofue Y, Okamoto S, et al. Selective ion sensors based on ionophore-modified graphene field-effect transistors. 2013;187:45-49. Copyright 2013, with permission from Elsevier. ${ }^{15}$

on sensitivity for receptor-ligand reactions. They utilized biotin and streptavidin for recognition, and functionalized $\mathrm{Si}$ nanowire-based FETs with biotin molecules. After introduction of $10 \mathrm{nM}$ streptavidin in $1 \times$ PBS, no change in the drain current was obtained. This is because most of streptavidin's intrinsic charge was screened by the solution $\left(\lambda_{\mathrm{D}} \sim 0.7 \mathrm{~nm}\right)$. When $0.1 \times$ PBS was used $\left(\lambda_{D} \sim 2.3 \mathrm{~nm}\right)$, the charge was partially screened. In $0.01 \times \operatorname{PBS}\left(\lambda_{\mathrm{D}} \sim 7.3 \mathrm{~nm}\right)$, adding $10 \mathrm{nM}$ of streptavidin resulted in a largely increased drain current. The majority of charge was unscreened in the solution, and the charge effectively modulated the band structure of the $\mathrm{Si}$ nanowire channel. The results indicate that the concentration of buffer solution is the indispensable factor for FET-based biosensors, and that the Debye length significantly affects the sensitivity of the sensors.

Some groups used buffer solution with significantly low concentration. ${ }^{12,20-22}$ Zheng et al ${ }^{20}$ demonstrated multiplexed electrical detection of cancer markers with Si nanowire-based sensor arrays (Figure 3A). The buffer solution was $1 \mu \mathrm{M}$ phosphate containing $2 \mu \mathrm{M}$ potassium chloride $(\mathrm{KCl})$ at a $\mathrm{pH}$ of 7.4. The Debye length of the buffer solution was estimated to be more than $100 \mathrm{~nm}$. To monitor three kinds of biomarkers, PSA, carcinoembryonic antigen (CEA), and mucin-1, the different Si nanowires were functionalized with antibodies for PSA, CEA, and mucin-1, respectively. The time dependences of the conductance in Si nanowire-based sensors were carried out after introduction of PSA, CEA, and mucin-1 in succession, resulting in multiplexed cancer marker detection with high sensitivity using the Si nanowirebased FETs. Moreover, it has been reported that real-time electrical detection of a single virus was demonstrated using Si nanowire-based FETs that were modified with antihemagglutinin for influenza A (Figure 3B). ${ }^{21}$ The virus is significantly larger than protein; thus, the experiments were carried out in $10 \mu \mathrm{M}$ PBS containing $10 \mu \mathrm{M} \mathrm{KCl}$ at a $\mathrm{pH}$ of 6.0 to obtain the long Debye length. When the virus bound to the 
A
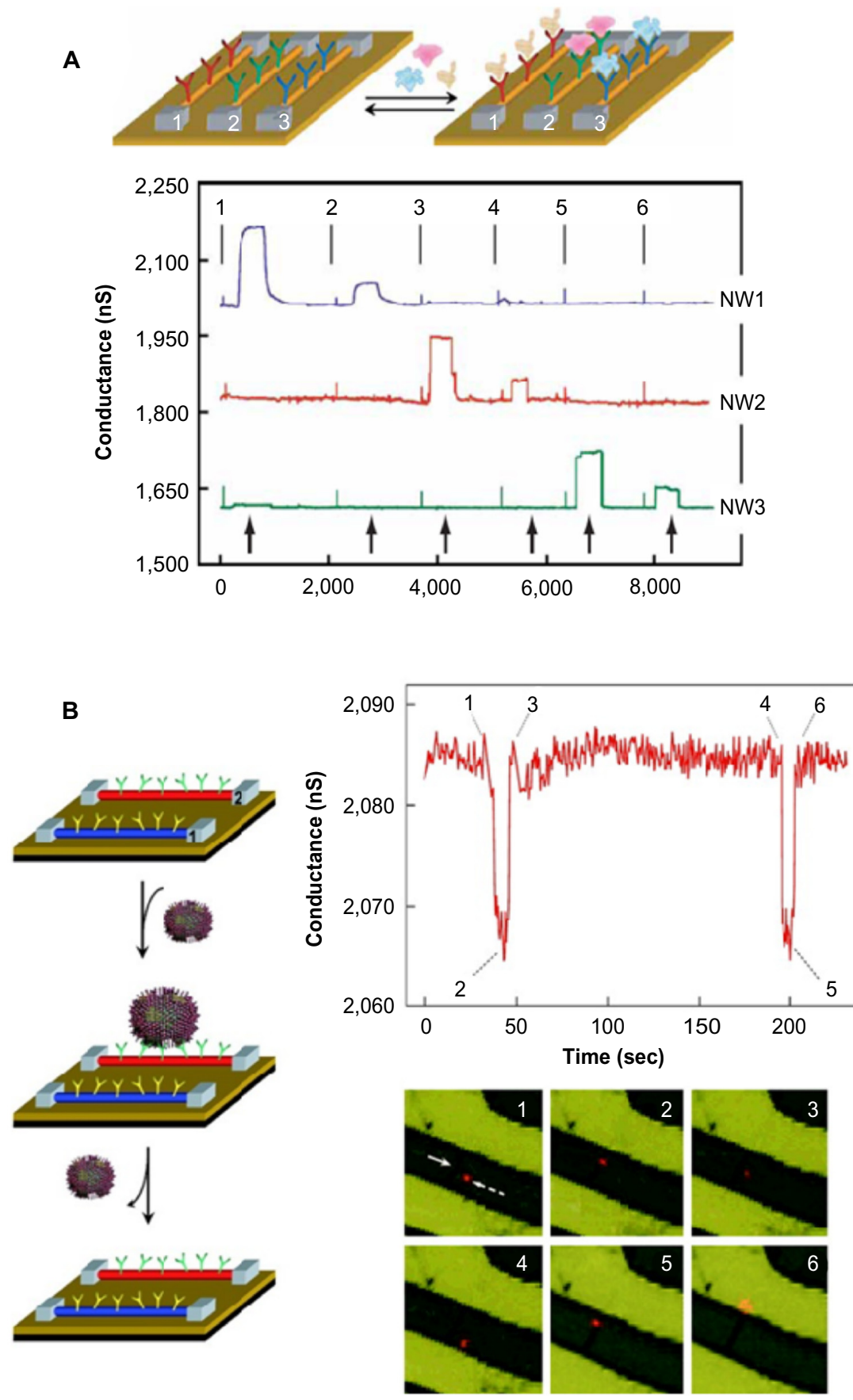

Figure 3 Electrical detection of biomolecules using buffer solution with low concentration.

Notes: (A) Multiplexed electrical detection of cancer markers with silicon (Si) nanowire (NW)-based sensor arrays. Prostate-specific antigen (PSA), carcinoembryonic antigen (CEA), and mucin-I were monitored using three different Si nanowires, which were functionalized with antibodies for PSA, CEA, and mucin-I, respectively. ${ }^{20}$ (B) Realtime electrical detections (upper right) of single virus using Si nanowire-based FETs. Optical images (lower right) using fluorescently labeled viruses supported the electrical measurements. ${ }^{21}$ The red dots in the image show virus, and the solid and dashed white arrows correspond to the positions of the Si nanowire and a single virus, respectively. ${ }^{21}$ Reprinted by permission from Macmillan Publishers Ltd: Nat Biotechnol. Zheng G, Patolsky F, Cui Y, et al. Multiplexed electrical detection of cancer markers with nanowire sensor arrays. 23:I294-130I, copyright 2005; ${ }^{20}$ Reprinted by permission from Macmillan Publishers Ltd: Proc Natl Acad Sci USA. Patolsky F, Zheng G, Hayden O, et al. Electrical detection of single viruses. I0I:I40I7-I4022. Copyright (2004) National Academy of Sciences, U.S.A. ${ }^{21}$

antibody, the conductance sharply decreased owing to the negative charge of the virus, and when the virus unbound, the conductance returned to the baseline. The optical images using fluorescently labeled viruses supported the electrical measurements. However, for practical uses, it is impossible to dilute only buffer solution, because body fluids such as blood, urine, and saliva contain a large amount of salts.

To overcome these issues, some researchers utilized receptors smaller than antibodies. The smaller receptors will combine with target molecules inside the Debye length. 
One of the most promising candidates is aptamers. ${ }^{23-26}$ They are artificial oligonucleic acid and bind to specific various target molecules such as lysozyme, thrombin, immunoglobulin (Ig)E, and PSA. Moreover, aptamers can be created in vitro. Hence, they are less expensive than antibodies but are very stable even at room temperature. The height of aptamers is approximately $2 \sim 3 \mathrm{~nm}$. So et a ${ }^{27}$ utilized deoxyribonucleic (DNA) aptamers as molecular recognition elements. Thrombin aptamers were immobilized on to single-walled carbon nanotubes (SWNTs) to detect thrombin. The decreases in conductance of CNTFETs were observed after thrombin solution was added, and thrombin concentration dependence was measured from $10 \mathrm{nM}$ to $300 \mathrm{nM}$. For detection of $\mathrm{IgE}$, IgE aptamers were anchored on to the SWNT or graphene channel using 1-pyrenebutanoic acid succinimidyl ester. ${ }^{28,29}$ The real-time measurements show that $\operatorname{IgE}$ in the range of $250 \mathrm{pM}$ to $160 \mathrm{nM}$ was effectively detected using CNTFETs and that the adsorption of IgE on to aptamers on SWNT channels follows the Langmuir adsorption isotherm (Figure 4A). ${ }^{30}$ The estimation of the dissociation constant results in a good affinity between IgE molecules and aptamers. ${ }^{31}$

The other candidate is antigen-binding fragment (Fab). ${ }^{32,33}$ As described, aptamers are useful receptors; however, they have not been created for many target molecules, such as cancer markers. On the other hand, Fab is a binding site of an antibody that can be created using enzyme papain in the laboratory (Figure 4B). ${ }^{13}$ Thus, Fab recognizes a specific antigen and is developed for detection of target molecules. The size of Fab is approximately $3 \sim 5 \mathrm{~nm}$. For this reason, binding events between Fab and target molecules occur within the electrical double layer in a buffer solution, and target molecules are expected to be detected with high sensitivity. Kim et $\mathrm{al}^{34}$ fabricated CNTFET-based biosensors that were functionalized with Fab for antigen-antibody reaction. They have reported that IgG was detected using Fab with higher sensitivity than using whole antibody. Okamoto et $\mathrm{al}^{13}$ have fabricated Fab-modified graphene FETs, and heat-shock proteins (HSPs) of the target biomolecules were detected (Figure 4B). The HSP concentration dependence showed that Fab and HSP have high affinity.

\section{Micro total analysis systems}

To perform real-time sensing of biomolecules based on nanomaterial transistors, target molecules are delivered into a vessel that is set on the transistors. The target molecules can be reacted to receptors that are functionalized with
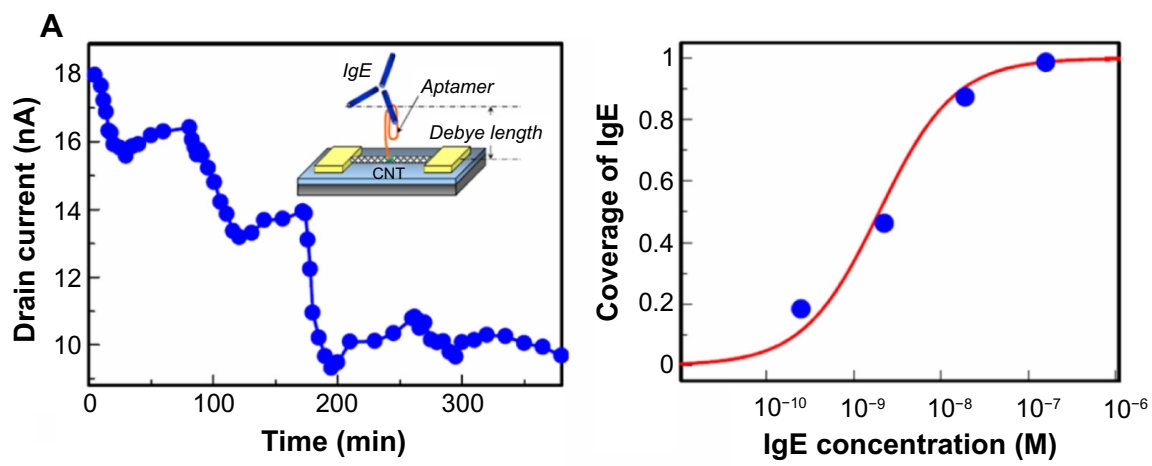

B
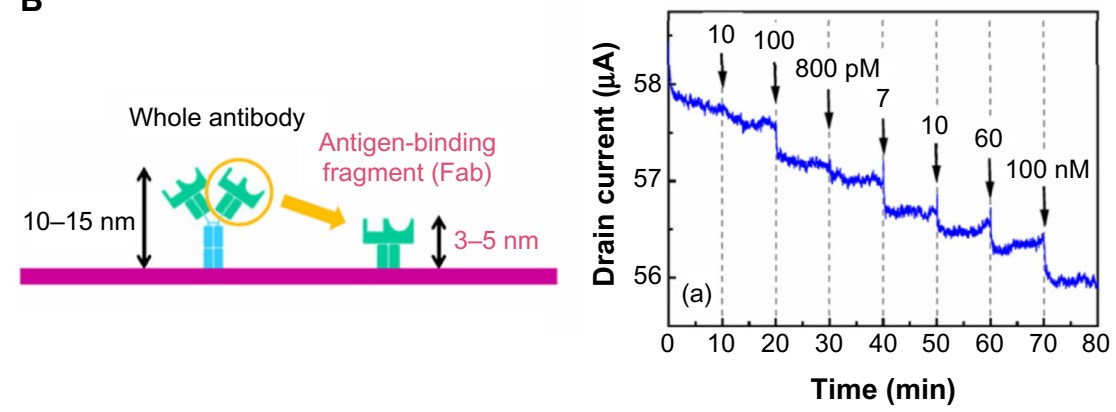

Figure 4 Electrical detection of biomolecules using small receptors.

Notes: (A) Real-time detection of immunoglobulin (Ig)E molecules in the range of $250 \mathrm{pM}$ to $160 \mathrm{nM}$ using aptamer-modified carbon nanotube (CNT) field-effect transistors (FETs) (left side). The adsorption of IgE on to aptamers on single-walled carbon nanotube channels follows the Langmuir adsorption isotherm (right side). ${ }^{30}$ (B) Schematic illustration of an antibody and an antigen-binding fragment (Fab) (left side) and heat-shock protein concentration dependence of a drain current in an Fab-modified graphene FET (right side). ${ }^{13}$ Reproduced with permission from Maehashi K and Matsumoto K. Label-free electrical detection using carbon nanotube-based biosensors. Sensors 2009;9:5368-5378. Copyright 2009 Multidisciplinary Digital Publishing Institute. ${ }^{30}$ Reproduced with permission from Okamoto S, Ohno Y, Maehashi K, et al. Immunosensors based on graphene field-effect transistors fabricated using antigen-binding fragment. Jpn J Appl Phys. 2012;51:06FD08. Copyright 20I2 The Japan Society of Applied Physics. ${ }^{13}$ 
nanomaterial channels. A simple method is to use a cell or a container. ${ }^{28,35-38}$ This cell or container is commonly made of Si rubber or Teflon and is directly placed on transistors. Solutions containing target molecules are dropped into the cell, and the molecules diffuse and reach receptors.

On the other hand, to realize hand-held biosensors based on FETs for home medical care systems, nanomaterial transistors are necessary to be integrated in a micro total analysis system ( $\mu$ TAS) in which several microdevices are integrated to perform diverse functions such as pretreatment, reaction, and detection. ${ }^{39-41}$ A simple $\mu$ TAS has microfluidic channels on sensing devices. Microfluidic systems need only micro- or nanoliter volumes of reagents in the microchannels, which allows rapid detection at low experimental cost. Microfluidic systems are usually made of poly(dimethylsiloxane) and are placed on nanomaterial-based sensor devices. ${ }^{42,43}$ The fluid flow velocity in the microchannel can be controlled by pumping systems. Tsujita et al ${ }^{44,45}$ fabricated microfluidic chips containing CNT-based sensors and pneumatic micropumps. Using integrated pneumatic micropumps, several reagents can be respectively introduced to arrayed sensors. As a result, several kinds of receptors can be automatically immobilized on to different CNTs, and simultaneous detection of several kinds of biomolecules can be performed. Recently, Chang et $\mathrm{al}^{46}$ demonstrated biomarker detection from human whole blood collected by a finger prick. They used polycarbonate membranes, and almost all blood cells and macroscopic impurities in whole blood were successfully removed. Thus, cancer biomarkers were effectively detected. These systems are indispensable techniques for hand-held biosensors in home medical care systems.

\section{Si nanowire-based biosensors}

In this section we introduce fabrication techniques of labelfree Si nanowire-based biosensors. As Si nanowires are $10 \sim 100 \mathrm{~nm}$ in diameter, they have a higher aspect ratio than conventional Si FETs. As a result, adsorption of biomolecules induces accumulation or depletion of carriers for entire regions of Si nanowires. Moreover, processes for fabrication of Si nanowire FETs are applicable to the conventional Si FET fabrication processes.

In general, Si nanowires can be created using two methods. One method is using bottom-up technology: ie, growth of Si nanowires using a metal catalyst by vapor-liquid-solid method (Figure 5A). The Si nanowires are scattered on $\mathrm{SiO}_{2} / \mathrm{Si}$ substrates. After formation of electrodes on the substrates, Si nanowire-based FETs can be fabricated. ${ }^{47}$ The method is suitable for creation of high-quality Si nanowires; however, it has disadvantages for controlling the length, diameter, and position of Si nanowires. The other method is top-down technology using $\mathrm{Si}$ on insulator substrates. $\mathrm{Si}$ nanowire channels can be formed by etching the Si layer on the insulator. The technology can fabricate position-controlled Si nanowires with the required length and diameter. Thus, the method is a promising candidate for large-scale mass production because complementary metal oxide semiconductor fabrication process can be applicable to the method. Si nanowires were formed using reactive ion etching, resulting in degraded device performance. ${ }^{48}$ To obtain high-quality $\mathrm{Si}$ nanowires by top-down technology, Stern et $\mathrm{al}^{36}$ have reported fabrication of Si nanowires without mobility degradation using ultrathin $\mathrm{Si}$ on insulator substrates and an anisotropic wet etching process (Figure 5B). They have detected mouse IgG and mouse IgA with $100 \mathrm{fM}$ for antimouse IgG- and IgAfunctionalized sensors based on complementary metal oxide semiconductor-compatible Si nanowires, respectively.

Typical fabricated Si nanowires are covered with $\mathrm{Si}$ oxide layers. Receptors such as antibodies are immobilized on the Si oxide surfaces. Two types of linkers are commonly used to bind the Si oxide surfaces to receptors. One is 3-(trimethoxysilyl) propyl aldehyde, which forms aldehyde groups to covalently immobilize receptors. ${ }^{20-22}$ The other is 3-aminopropyltriethoxysilane (APTES), which provides surfaces with amino groups. APTES was used to immobilize antibody or DNA probes. ${ }^{12,19}$

\section{Nanocarbon-based biosensors}

In the past decade, chemical and biological sensors based on nanocarbon materials, such as CNTs and graphene, have attracted considerable attention owing to their nanometer size and highly specific surface area. As compared with Si-based sensors, CNT and graphene FETs will operate in a solution without any insulators on channels, because CNTs and graphene have high potential window and chemical stability in solution. Furthermore, every atom in an SWNT or monolayer graphene is located on the surface. Thus, recognition events are expected to directly occur on the surface, leading to extreme sensitivity to the surrounding environments.

\section{CNTs}

CNTs are formed as a sheet of carbon atoms rolled up into tubes. Mutiwalled carbon nanotubes (MWNTs) are made up of several concentric cylindrical sheets with a diameter of 2-100 nm. On the other hand, SWNTs consist of a single layer of carbon atoms with a diameter of 0.4-2 nm. The electronic structure of SWNTs can be determined by the $(n, m)$ 
A
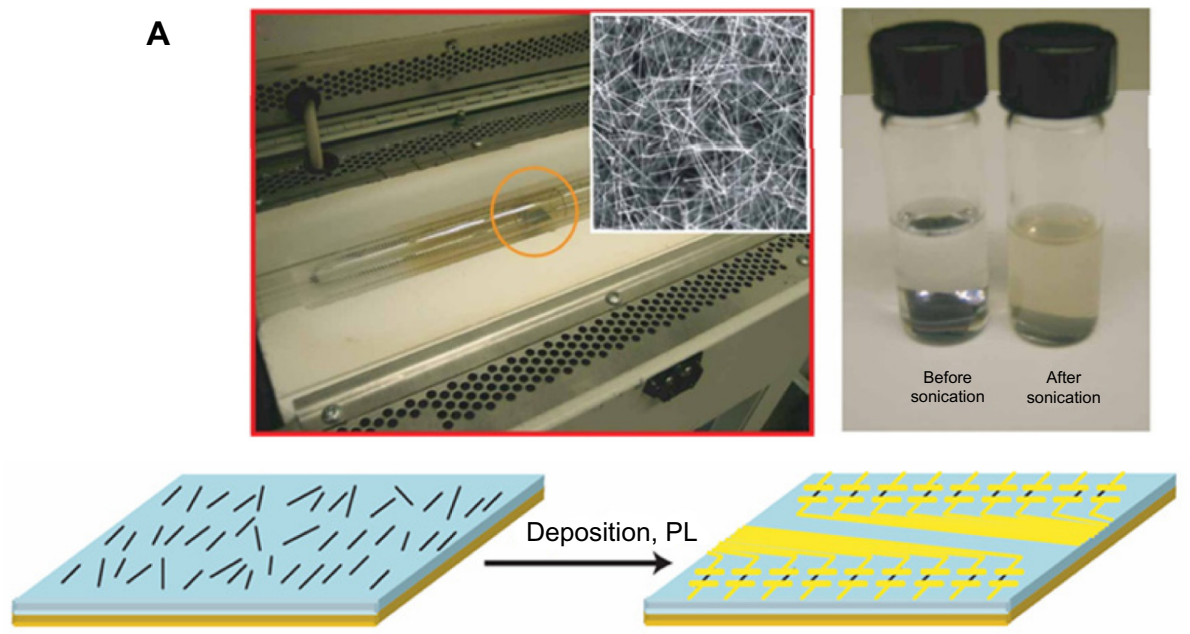

B
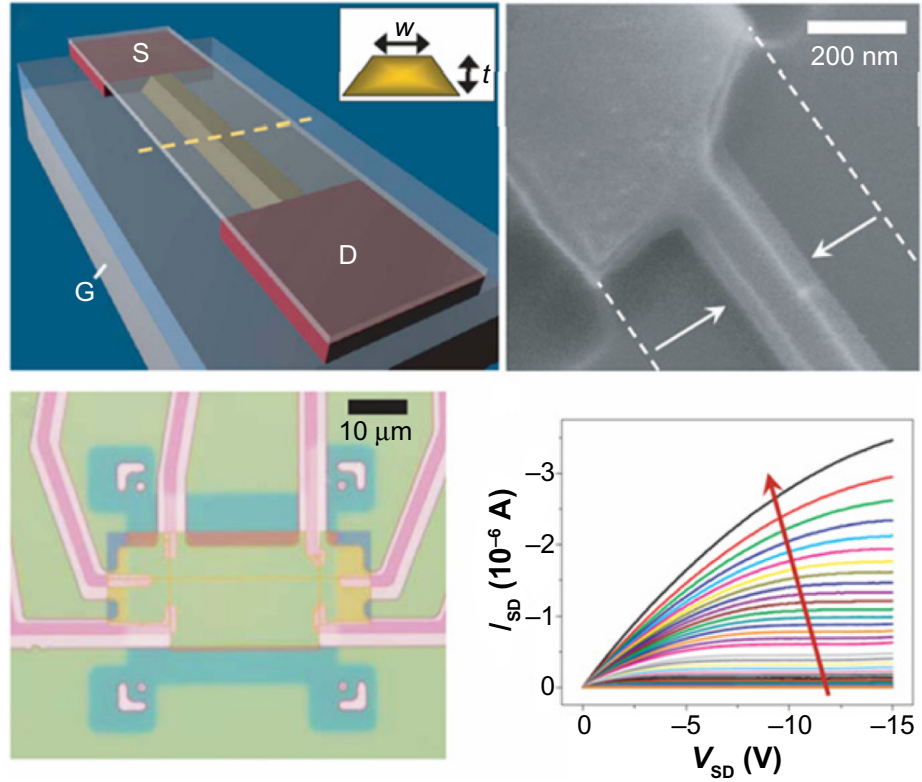

Figure 5 Silicon nanowire-based biosensors.

Notes: (A) Fabrication of silicon (Si) nanowire field-effect transistors (FETs) using bottom-up technology. The Si nanowires are grown using metal catalyst by vapor-liquidsolid method. Then, the $\mathrm{Si}$ nanowires are scattered on silicon dioxide $\left(\mathrm{SiO}_{2}\right) / \mathrm{Si}$ substrates. After formation of electrodes on the substrates, Si nanowire-based FETs can be fabricated. ${ }^{47}$ (B) Fabrication of Si nanowire FETs using ultrathin Si on insulator substrates and anisotropic wet etching process. ${ }^{36}$ Reprinted by permission from Macmillan Publishers Ltd: Nature Protoc. Patolsky F, Zheng G, Lieber CM. Fabrication of silicon nanowire devices for ultrasensitive, label-free, real-time detection of biological and chemical species. I:I7II-1724. Copyright 2006; ${ }^{47}$ Reprinted by permission from Macmillan Publishers Ltd: Nature. Stern E, Klemic JF, Routenberg DA, et al. Label-free immunodetection with CMOS-compatible semiconducting nanowires.2007;445:519-522. Copyright 2007.36

Abbreviations: S, source; D, drain; G, gate.

chiral indices. ${ }^{49}$ Depending on the nanotube symmetry and diameter, two-thirds of $(n, m)$ classes of SWNTs are expected to be semiconducting; the other being metallic. When semiconducting SWNTs are used as channels, CNTFETs can be fabricated..$^{50,51}$

There are two classical types for the structures of CNTFETs. In the first type, one or a few SWNTs are bridged between source and drain electrodes to obtain high mobility and on/off ratio. For fabricating the device, positioncontrolled growth is commonly utilized (Figure 6A). ${ }^{52,53}$ First, a patterned catalyst is formed by a photolithography and liftoff process on heavily doped Si substrates covered with an $\mathrm{SiO}_{2}$ layer. SWNTs are grown by chemical vapor deposition (CVD). Source and drain electrodes are fabricated on the patterned catalyst. In air, the CNTFETs show p-type characteristics. The second type is to use a random network of SWNTs. As the random network configuration consists of both metallic and semiconducting SWNTs, the density of SWNTs and the length between source and drain electrodes are necessarily controlled. This method is useful for mass production because larger drain currents and higher uniformity of electrical properties are easy obtained. Many groups 


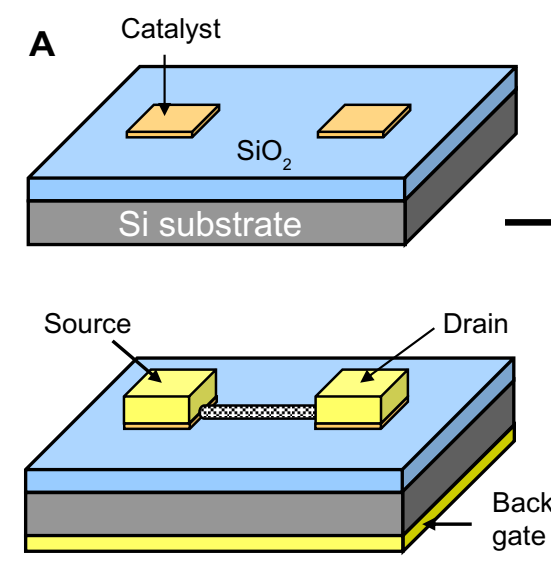

B

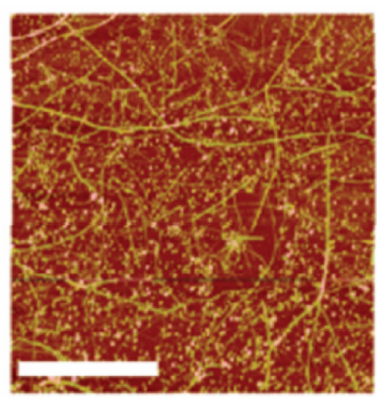

$2 \mu \mathrm{m}$

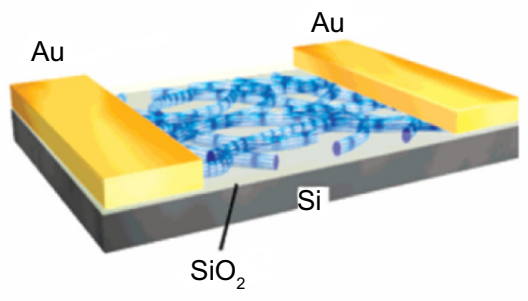

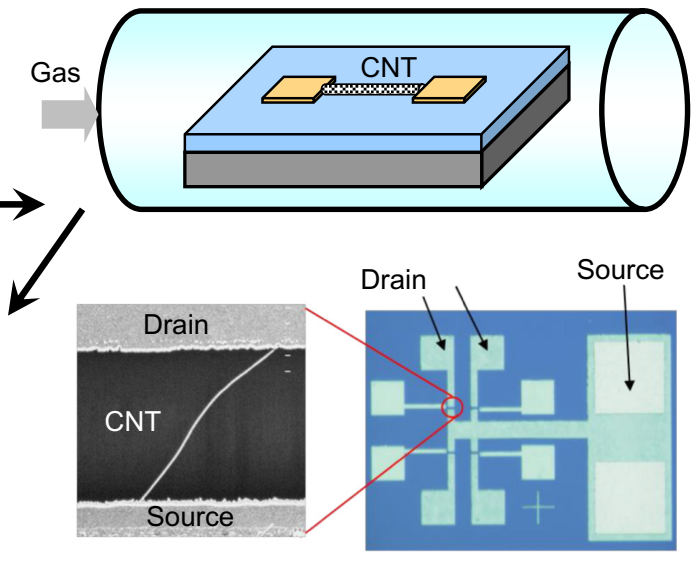

C
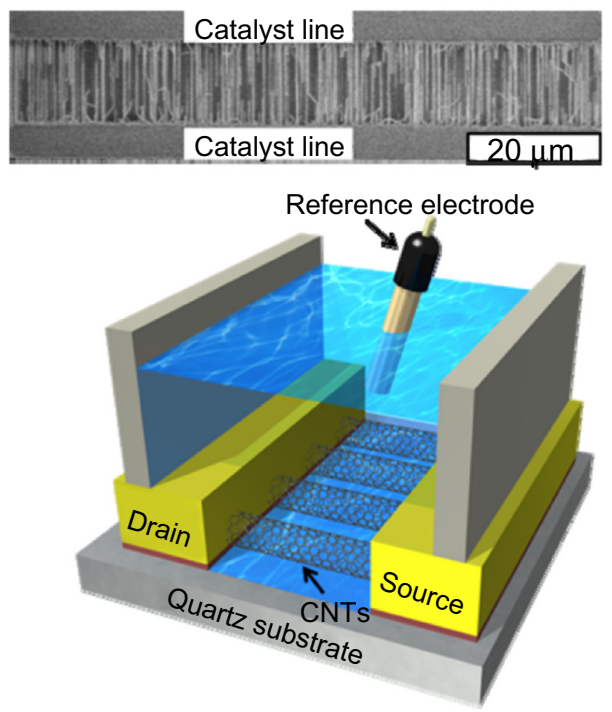

Figure 6 Carbon nanotube (CNT)-based biosensors.

Notes: (A) Position-controlled growth of single-walled carbon nanotubes (SWNTs) by patterned catalyst for fabrication of carbon nanotube field-effect transistors (FETs). First, a patterned catalyst is formed by photolithography and liftoff process on silicon ( $\mathrm{Si}$ ) substrates covered with a silicon dioxide ( $\mathrm{SiO}_{2}$ ) layer. SWNTs are grown by chemical vapor deposition. Source and drain electrodes are fabricated on the patterned catalyst. Optical and scanning electron microscope (SEM) images of CNTFETs are shown. (B) Atomic force microscopy image of an SWNT network and schematic illustration of a network CNTFET. ${ }^{56}$ (C) SEM image of horizontally aligned SWNTs between catalyst lines synthesized on quartz substrate and a schematic illustration of electrolyte-gated CNTFET sensor. ${ }^{60}$ Reprinted with permission from Gui EL, Li L-J, Zhang K, et al. DNA sensing by field-effect transistors based on networks of carbon nanotubes. J Am Chem Soc. I29: 14427-14432. Copyright 2007 American Chemical Society. ${ }^{56}$ Reprinted with permission from Okuda S, Okamoto S, Ohno Y, et al. Horizontally aligned carbon nanotubes on a quartz substrate for chemical and biological sensing. J Phys Chem C. 1 16:19490-19495. Copyright 2012 American Chemical Society. ${ }^{60}$

have utilized network SWNTs as FETs channels for biosensing (Figure 6B). ${ }^{35,54-57}$ In recent years, controlled growth of SWNTs by surface-guided growth on single crystalline substrates such as quartz or sapphire has attracted much attention. ${ }^{58,59}$ Okuda et $\mathrm{al}^{60}$ reported electrolyte-gated sensors based on an FET consisting of horizontally aligned SWNTs synthesized on single crystal quartz (Figure 6C).

For selective detection of biomolecules, receptors are required to be immobilized on SWNT channels. Selecting appropriate molecules that link SWNT channels to receptors is indispensable. If the molecules induce defects into the SWNT channels, performance of the devices will be degraded. One of the suitable molecules is 1-pyrenebutanoic acid succinimidyl ester, which is used as a bifunctional linker. ${ }^{61}$ The pyrenyl group of the linker strongly interacts with the six-membered rings of SWNTs via $\pi$-stacking. As a result, the linker can be noncovalently functionalized with SWNTs without degradation of the device performance. The succinimidyl ester of the linker, which is anchored on to SWNTs, reacts with the amino groups of receptors to form amide bonds for functionalization. Li et $\mathrm{al}^{35}$ functionalized antibodies on SWNTs using 1-pyrenebutanoic acid succinimidyl ester and detected PSA with high sensitivity. Kim et al $^{38}$ enhanced sensitivity of CNTFET-based sensors by controlling density of antibody using 1-pyrenebutanoic acid succinimidyl ester. 


\section{Graphene}

Graphene has atomic layers of carbon atoms tightly packed into a two-dimensional honeycomb lattice. ${ }^{62}$ It has been known for its excellent characteristics, such as mechanical and electronic properties. ${ }^{63-65}$ The extraordinary properties of grapheme, such as high carrier mobility, have tremendous potential in its application. ${ }^{66,67}$ When monolayer graphene with high quality is incorporated as a channel of FETs, high carrier mobility will be expected. Thus, a change in tiny charges owing to adsorption or desorption of analyte on the graphene channel in a solution will be detected as a large change in the conductance of the device. ${ }^{68}$ Therefore, graphene FETs will be used as electric readout biological or chemical sensors.

There are several techniques to obtain graphene. The first technique is to mechanically exfoliate graphene using an adhesive tape to peel thin flakes from bulk graphite. ${ }^{69}$ Then, the tape with graphite film is adhered to an $\mathrm{SiO}_{2} / \mathrm{Si}$ substrate. After removing the tape, graphene is found on the substrate. The method is suitable for obtaining high-quality graphene. Some groups developed chemical and biological sensors based on graphene FETs using the mechanical exfoliation technique (Figure 7A) ${ }^{29,37,70,71}$ However, it is impossible to control the size or location of the graphene. For simple mass production of graphene, chemical reduction of graphene oxide was developed..$^{72}$ Graphene oxide is chemically exfoliated from bulk graphite under strongly acidic conditions. Mohanty and Berry ${ }^{73}$ fabricated a single bacterium resolution interfacial device based on chemically modified graphene. Mao et al ${ }^{74}$ specifically detected $\operatorname{IgG}$ using a reduced graphene oxide sheet (Figure 7B). Another technique is to eliminate $\mathrm{Si}$ atoms from the surface of single crystal $\mathrm{SiC}$ substrates heated at above $1,200^{\circ} \mathrm{C}$ in an ultrahigh vacuum. ${ }^{75,76}$ Ang et al ${ }^{77}$ demonstrated $\mathrm{pH}$ sensors based on epitaxial graphene. Recently, CVD on metal substrates has been intensively investigated as an attractive method to synthesize graphene. ${ }^{78,79} \mathrm{Nickel}(\mathrm{Ni})$ and copper $(\mathrm{Cu})$ are commonly used as metal substrates. For growth on Ni substrates, graphene is synthesized at cooling stages. ${ }^{80}$ On the other hand, graphene grows on $\mathrm{Cu}$ substrates by surface adsorption of carbon atoms. ${ }^{81}$ For electronic applications such as sensors, existing graphene layer grown using CVD needs a transfer process from a metal substrate to insulating substrates. Recently, transfer processes using various different methods have been performed. Some groups fabricated chemical and
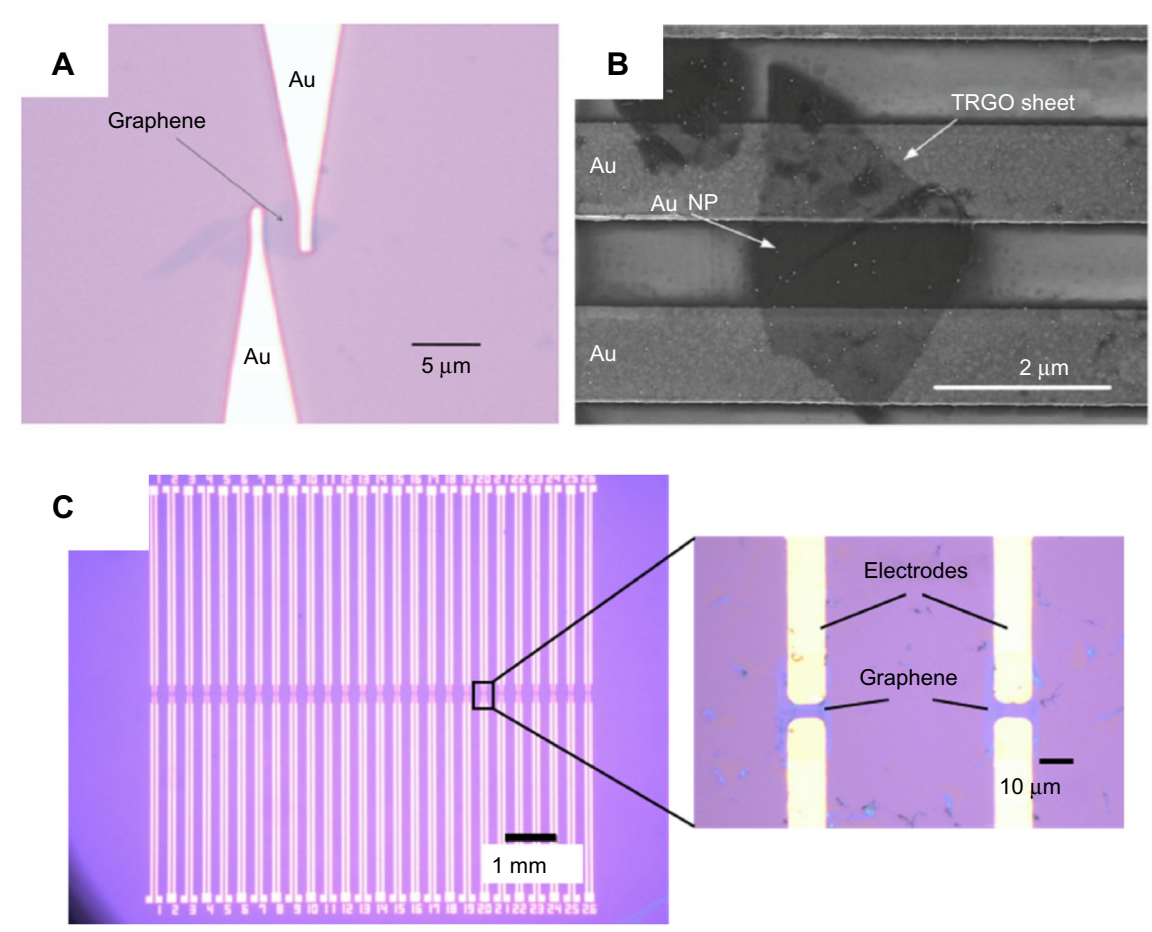

Figure 7 Graphene-based biosensors.

Notes: (A) Optical image of a graphene field-effect transistor (FET). The graphene was obtained by mechanical exfoliation. ${ }^{37}$ (B) Scanning electron microscope image of a thermally reduced graphene oxide (TRGO) sheet with gold (Au) nanoparticle (NP)-antibody conjugates spanning across Au electrodes. ${ }^{74}$ (C) Optical image of graphene-FET array. The graphene was synthesized by chemical vapor deposition. ${ }^{84}$ Reprinted with permission from Ohno Y, Maehashi K, Yamashiro Y, et al. Electrolyte-gated graphene field-effect transistors for detecting ph and protein adsorption. Nano Lett. 9:3318-3322. Copyright 2009 American Chemical Society. ${ }^{37}$ Reprinted from Mao S, Lu G, Yu K, et al. Specific protein detection using thermally reduced graphene oxide sheet decorated with gold nanoparticle-antibody conjugates. Adv Mater. 22:352I-3526. Copyright $(\underset{1}{ }$ 2010 WILEY-VCH Verlag GmbH \& Co. KGaA, Winheim. ${ }^{74}$ Reprinted from Zaifuddin NM, Okamoto S, Ikuta T, et al. pH sensor based on chemical-vapor-depositionsynthesized graphene transistor array. Jpn J Appl Phys. 52:06GK04. Copyright 2013 The Japan Society of Applied Physics. ${ }^{84}$ 
biological sensors using CVD-graphene FETs (Figure 7C). ${ }^{82-}$

${ }^{84}$ Park et $\mathrm{al}^{85}$ demonstrated multiplexed $\mathrm{pH}$ sensing using monolithic FETs based on CVD graphene.

\section{Conclusion}

In this review we provide an introduction to label-free biosensors based on nanomaterial transistors using $\mathrm{Si}$ nanowires, CNTs, and graphene. The nanomaterial transistors can detect tiny charges of target molecules, as binding events between receptors and target molecules induce accumulation or depletion of carriers for entire regions of the nanomaterials. The concentration of buffer solution is the indispensable factor for FET-based biosensors, and the Debye length significantly affects the sensitivity of the sensors. Thus, reports were introduced that a buffer solution with low concentration was used to increase the Debye length, and that small receptors such as aptamer and Fab were utilized to obtain high sensitivity.

Label-free biosensors based on nanomaterial transistors are still developing and are necessary to overcome several difficulties for commercialization, such as reliability, reproducibility, and mass production methods for the biosensors. We believe that label-free nanomaterial-based biosensors are a promising candidate for the development of hand-held multiplex biosensors in a home medical care system.

\section{Disclosure}

The authors report no conflicts of interest in this work.

\section{References}

1. Lyon LA, Musick MD, Natan MJ. Colloidal au-enhanced surface plasmon resonance immunosensing. Anal Chem. 1998;70: 5177-5183.

2. Nice EC, Catimel B. Instrumental biosensors: new perspectives for the analysis of biomolecular interactions. Bioessays. 1999;21:339-352.

3. Tess ME, Cox JA. Chemical and biochemical sensors based on advances in materials chemistry. J Pharm Biomed Anal. 1999;19:55-68.

4. Brekkan E, Lundqvist A, Lundahl P. Immobilized membrane vesicle or proteoliposome affinity chromatography. Frontal analysis of interactions of cytochalasin b and d-glucose with the human red cell glucose transporter. Biochemistry. 1996;35:12141-12145.

5. Willardson BM, Wilkins JF, Rand TA, et al. Development and testing of a bacterial biosensor for toluene-based environmental contaminants. Appl Environ Microbiol. 1998;64:1006-1012.

6. Okuno J, Maehashi K, Kerman K, et al. Label-free immunosensor for prostate-specific antigen based on single-walled carbon nanotube arraymodified microelectrodes. Biosens Bioelectron. 2007;22:2377-2381.

7. Drummond TG, Hill MG, Barton JK. Electrochemical DNA sensors. Nat Biotechnol. 2003;21:1192-1199.

8. Cheng AKH, Ge B, Yu H-Z. Aptamer-based biosensors for label-free voltammetric detection of lysozyme. Anal Chem. 2007;79:5158-5164.

9. Daniels JS, Pourmand N. Label-free impedance biosensors: opportunities and challenges. Electroanalysis. 2007;19:1239-1257.

10. Macdonald DD. Reflections on the history of electrochemical impedance spectroscopy. Electrochim Acta. 2006;51:1376-1388.

11. Minot ED, Janssens AM, Heller I, et al. Carbon nanotube biosensors: the critical role of the reference electrode. Appl Phys Lett. 2007;91:093507.
12. Kim A, Ah CS, Yu HY, et al. Ultrasensitive, label-free, and real-time immunodetection using silicon field-effect transistors. Appl Phys Lett. 2007;91:103901.

13. Okamoto S, Ohno Y, Maehashi K, et al. Immunosensors based on graphene field-effect transistors fabricated using antigen-binding fragment. Jpn J Appl Phys. 2012;51:06FD08.

14. Novoselov KS, Geim AK, Morozov SV, et al. Electric field effect in atomically thin carbon films. Science. 2004;306:666-669.

15. Maehashi K, Sofue Y, Okamoto S, et al. Selective ion sensors based on ionophore-modified graphene field-effect transistors. Sensors Actuators B: Chem. 2013;187:45-49.

16. Debye P. Dieletric properties of pure liquids. Chem Rev. 1936;19: 171-182.

17. Rudikoff S, Potter M. Size differences among immunoglobulin heavy chains from phosphorylcholine-binding proteins. Proc Natl Acad Sci US A. 1976;73:2109-2112.

18. Teillaud J, Desaymard C, Giusti A, et al. Monoclonal antibodies reveal the structural basis of antibody diversity. Science. 1983;222: 721-726.

19. Stern E, Wagner R, Sigworth FJ, et al. Importance of the debye screening length on nanowire field effect transistor sensors. Nano Lett. 2007; 7:3405-3409.

20. Zheng G, Patolsky F, Cui Y, et al. Multiplexed electrical detection of cancer markers with nanowire sensor arrays. Nat Biotechnol. 2005;23:1294-1301.

21. Patolsky F, Zheng G, Hayden O, et al. Electrical detection of single viruses. Proc Natl Acad Sci U S A. 2004;101:14017-14022.

22. Wang WU, Chen C, Lin K-h, et al. Label-free detection of smallmolecule-protein interactions by using nanowire nanosensors. Proc Natl Acad Sci U S A. 2005;102:3208-3212.

23. Tuerk C, Gold L. Systematic evolution of ligands by exponential enrichment: RNA ligands to bacteriophage T4 DNA polymerase. Science. 1990;249:505-510.

24. Robertson DL, Joyce GF. Selection in vitro of an rna enzyme that specifically cleaves single-stranded DNA. Nature. 1990;344:467-468.

25. Ellington AD, Szostak JW. In vitro selection of rna molecules that bind specific ligands. Nature. 1990;346:818-822.

26. O'Sullivan C. Aptasensors: the future of biosensing? Anal Bioanal Chem. 2002;372:44-48.

27. So H-M, Won K, Kim YH, et al. Single-walled carbon nanotube biosensors using aptamers as molecular recognition elements. J Am Chem Soc. 2005;127:11906-11907.

28. Maehashi K, Katsura T, Kerman K, et al. Label-free protein biosensor based on aptamer-modified carbon nanotube field-effect transistors. Anal Chem. 2007;79:782-787.

29. Ohno Y, Maehashi K, Matsumoto K. Label free biosensors based on aptamer modified graphene field effect transistors. J Am Chem Soc. 2010;132:18012-18013.

30. Maehashi K, Matsumoto K. Label-free electrical detection using carbon nanotube-based biosensors. Sensors. 2009;9:5368-5378.

31. Maehashi K, Matsumoto K, Takamura Y, et al. Aptamer-based label-free immunosensors using carbon nanotube field-effect transistors. Electroanalysis. 2009;21:1285-1290.

32. Pilz I, Schwarz E, Palm W. Small-angle X-ray studies of the fab and fc fragments from the human immunoglobulin molecule kol. Eur $J$ Biochem. 1976;71:239-247.

33. Ng PC, Osawa Y. Preparation and characterization of the fab and F(ab') fragments of an aromatase activity-suppressing monoclonal antibody. Steroids. 1997;62:776-781.

34. Kim JP, Lee BY, Hong S, et al. Ultrasensitive carbon nanotube-based biosensors using antibody-binding fragments. Anal Biochem. 2008;381: 193-198.

35. Li C, Curreli M, Lin H, et al. Complementary detection of prostatespecific antigen using In2O3 nanowires and carbon nanotubes. $J \mathrm{Am}$ Chem Soc. 2005;127:12484-12485.

36. Stern E, Klemic JF, Routenberg DA, et al. Label-free immunodetection with CMOS-compatible semiconducting nanowires. Nature. 2007;445:519-522. 
37. Ohno Y, Maehashi K, Yamashiro Y, et al. Electrolyte-gated graphene field-effect transistors for detecting ph and protein adsorption. Nano Lett. 2009;9:3318-3322.

38. Kim JP, Lee BY, Lee J, et al. Enhancement of sensitivity and specificity by surface modification of carbon nanotubes in diagnosis of prostate cancer based on carbon nanotube field effect transistors. Biosens Bioelectron. 2009;24:3372-3378.

39. Reyes DR, Iossifidis D, Auroux P-A, et al. Micro total analysis systems. 1. Introduction, theory, and technology. Anal Chem. 2002;74: 2623-2636.

40. Manz A, Graber N, Widmer HM. Miniaturized total chemical analysis systems: a novel concept for chemical sensing. Sensors Actuators B: Chem. 1990;1:244-248.

41. Harrison DJ, Fluri K, Seiler K, et al. Micromachining a miniaturized capillary electrophoresis-based chemical analysis system on a chip. Science. 1993;261:895-897.

42. Hahm J-I, Lieber CM. Direct ultrasensitive electrical detection of DNA and DNA sequence variations using nanowire nanosensors. Nano Lett. 2003;4:51-54.

43. He RX, Lin P, Liu ZK, et al. Solution-gated graphene field effect transistors integrated in microfluidic systems and used for flow velocity detection. Nano Lett. 2012;12:1404-1409.

44. Tsujita Y, Maehashi K, Matsumoto K, et al. Carbon nanotube amperometric chips with pneumatic micropumps. Jpn J Appl Phys. 2008;47:2064-2067.

45. Tsujita Y, Maehashi K, Matsumoto K, et al. Microfluidic and label-free multi-immunosensors based on carbon nanotube microelectrodes. Jpn J Appl Phys. 2009;48:06FJ02.

46. Chang H-K, Ishikawa FN, Zhang R, et al. Rapid, label-free, electrical whole blood bioassay based on nanobiosensor systems. ACS Nano. 2011;5:9883-9891.

47. Patolsky F, Zheng G, Lieber CM. Fabrication of silicon nanowire devices for ultrasensitive, label-free, real-time detection of biological and chemical species. Nature Protoc. 2006;1:1711-1724.

48. Li Z, Chen Y, Li X, et al. Sequence-specific label-free DNA sensors based on silicon nanowires. Nano Lett. 2004;4:245-247.

49. Saito R, Fujita M, Dresselhaus G, et al. Electronic structure of chiral graphene tubules. Appl Phys Lett. 1992;60:2204-2206.

50. Tans SJ, Verschueren ARM, Dekker C. Room-temperature transistor based on a single carbon nanotube. Nature. 1998;393:49-52.

51. Martel R, Schmidt T, Shea HR, et al. Single- and multi-wall carbon nanotube field-effect transistors. Appl Phys Lett. 1998;73: 2447-2449.

52. Kong J, Soh HT, Cassell AM, et al. Synthesis of individual singlewalled carbon nanotubes on patterned silicon wafers. Nature. 1998;395: 878-881.

53. Kaminishi D, Ozaki H, Ohno Y, et al. Air-stable n-type carbon nanotube field-effect transistors with $\mathrm{Si}_{3} \mathrm{~N}_{4}$ passivation films fabricated by catalytic chemical vapor deposition. Appl Phys Lett. 2005;86:113115.

54. Chen RJ, Bangsaruntip S, Drouvalakis KA, et al. Noncovalent functionalization of carbon nanotubes for highly specific electronic biosensors. Proc Natl Acad Sci U S A. 2003;100:4984-4989.

55. Star A, Gabriel J-CP, Bradley K, et al. Electronic detection of specific protein binding using nanotube fet devices. Nano Lett. 2003;3: 459-463.

56. Gui EL, Li L-J, Zhang K, et al. DNA sensing by field-effect transistors based on networks of carbon nanotubes. J Am Chem Soc. 2007;129: $14427-14432$

57. So H-M, Park D-W, Jeon E-K, et al. Detection and titer estimation of escherichia coli using aptamer-functionalized single-walled carbon-nanotube field-effect transistors. Small. 2008;4:197-201.

58. Han S, Liu X, Zhou C. Template-free directional growth of single-walled carbon nanotubes on a- and r-plane sapphire. J Am Chem Soc. 2005; 127:5294-5295.

59. Kang SJ, Kocabas C, Ozel T, et al. High-performance electronics using dense, perfectly aligned arrays of single-walled carbon nanotubes. Nat Nanotechnol. 2007;2:230-236.
60. Okuda S, Okamoto S, Ohno Y, et al. Horizontally aligned carbon nanotubes on a quartz substrate for chemical and biological sensing. J Phys Chem C 2012;116:19490-19495.

61. Chen RJ, Zhang Y, Wang D, et al. Noncovalent sidewall functionalization of single-walled carbon nanotubes for protein immobilization. J Am Chem Soc. 2001;123:3838-3839.

62. Geim AK, Novoselov KS. The rise of graphene. Nat Mater. 2007;6: 183-191.

63. Balandin AA, Ghosh S, Bao W, et al. Superior thermal conductivity of single-layer graphene. Nano Lett. 2008;8:902-907.

64. Bolotin K, Sikes K, Jiang Z, et al. Ultrahigh electron mobility in suspended graphene. Solid State Commun. 2008;146:351-355.

65. Nair RR, Blake P, Grigorenko AN, et al. Fine structure constant defines visual transparency of graphene. Science. 2008;320:1308.

66. Lin Y-M, Jenkins KA, Valdes-Garcia A, et al. Operation of graphene transistors at gigahertz frequencies. Nano Lett. 2009;9:422-426.

67. Liao L, Lin YC, Bao M, et al. High-speed graphene transistors with a self-aligned nanowire gate. Nature. 2010;467:305-308.

68. Schedin F, Geim AK, Morozov SV, et al. Detection of individual gas molecules adsorbed on graphene. Nat Mater. 2007;6:652-655.

69. Novoselov KS, Geim AK, Morozov SV, et al. Two-dimensional gas of massless dirac fermions in graphene. Nature. 2005;438: 197-200.

70. Sofue Y, Ohno Y, Maehashi K, et al. Highly sensitive electrical detection of sodium ions based on graphene field-effect transistors. Jpn J Appl Phys. 2011;50:06GE07.

71. Cheng Z, Li Q, Li Z, et al. Suspended graphene sensors with improved signal and reduced noise. Nano Lett. 2010;10:1864-1868.

72. Gómez-Navarro C, Weitz RT, Bittner AM, et al. Electronic transport properties of individual chemically reduced graphene oxide sheets. Nano Lett. 2007;7:3499-3503.

73. Mohanty N, Berry V. Graphene-based single-bacterium resolution biodevice and DNA transistor: interfacing graphene derivatives with nanoscale and microscale biocomponents. Nano Lett. 2008;8: 4469-4476.

74. Mao S, Lu G, Yu K, et al. Specific protein detection using thermally reduced graphene oxide sheet decorated with gold nanoparticle-antibody conjugates. Adv Mater. 2010;22:3521-3526.

75. Ohta T, Bostwick A, Seyller T, et al. Controlling the electronic structure of bilayer graphene. Science. 2006;313:951-954.

76. Berger C, Song Z, Li X, et al. Electronic confinement and coherence in patterned epitaxial graphene. Science. 2006;312:1191-1196.

77. Ang PK, Chen W, Wee AT, et al. Solution-gated epitaxial graphene as ph sensor. J Am Chem Soc. 2008;130:14392-14393.

78. Reina A, Jia X, Ho J, et al. Large area, few-layer graphene films on arbitrary substrates by chemical vapor deposition. Nano Lett. 2008;9:30-35

79. Li X, Cai W, Colombo L, et al. Evolution of graphene growth on $\mathrm{Ni}$ and $\mathrm{Cu}$ by carbon isotope labeling. Nano Lett. 2009;9:4268-4272.

80. Yu Q, Lian J, Siriponglert S, et al. Graphene segregated on Ni surfaces and transferred to insulators. Appl Phys Lett. 2008;93:113103-113103.

81. Li X, Cai W, An J, et al. Large-area synthesis of high-quality and uniform graphene films on copper foils. Science. 2009;324: 1312-1314

82. Huang Y, Dong X, Shi Y, et al. Nanoelectronic biosensors based on CVD grown graphene. Nanoscale. 2010;2:1485-1488.

83. Kwak YH, Choi DS, Kim YN, et al. Flexible glucose sensor using CVD-grown graphene-based field effect transistor. Biosens Bioelectron. 2012;37:82-87.

84. Zaifuddin NM, Okamoto S, Ikuta T, et al. pH sensor based on chemicalvapor-deposition-synthesized graphene transistor array. Jpn $\mathrm{J} \mathrm{Appl}$ Phys. 2013;52:06GK04.

85. Park J-U, Nam S, Lee M-S, et al. Synthesis of monolithic graphenegraphite integrated electronics. Nat Mater. 2012;11:120-125. 
Nanobiosensors in Disease Diagnosis

Dovepress

\section{Publish your work in this journal}

Nanobiosensors in Disease Diagnosis is an international, peer-reviewed, open access journal publishing original research, reports, reviews and commentaries including but not confined to: Diagnosis of diseases including cancer, cardiovascular, infectious diseases; Molecular modeling in diagnosis; Enzyme and membrane technologies; and quantum dot fluorescence technologies for monitoring toxins and pathogens. The manuscript management system is completely online and includes a very quick and fair peer-review system, which is all easy to use. Visit $\mathrm{http}: / /$ www.dovepress.com/testimonials.php to read real quotes from published authors.

Submit your manuscript here: http://www.dovepress.com/nanobiosensors-in-disease-diagnosis-journal 